\title{
Reconstruction of the in-plane mode shape of a rotating tire with a continuous scanning measurement using the Hilbert -Huang Transform
}

\author{
Jongsuh Lee ${ }^{1)}$, Semyung Wang ${ }^{1, a)}$, Bert Pluymers ${ }^{2)}$, Wim Desmet ${ }^{2)}$ and Peter Kindt ${ }^{3)}$ \\ ${ }^{1}$ School of Mechatronics, Gwangju Institute of Science and Technology, Gwangju, 500-712, Korea \\ ${ }^{2}$ Katholieke Universiteit Leuven, Leuven, Belgium \\ ${ }^{3}$ Goodyear Innovation Center, Colmar-Berg, Luxembourge \\ a)Corresponding Author. Email address: smwang@gist.ac.kr \\ Telephone: $+82-62-715-2390$ \\ Fax: $+82-62-715-2384$
}

Generally, the dynamic characteristics (natural frequency, damping and mode shape) of a structure can be estimated by experimental modal analysis (EMA). Among these dynamic characteristics, mode shape requires multiple measurements of the structure at different positions, which increases the experimental cost and time. Recently, the Hilbert-Huang transform (HHT) method has been introduced to extract mode-shape information from a continuous measurement, which requires vibration measurements from one position to another position continuously with a non-contact sensor. In this research study, an effort has been made to estimate the mode shapes of a rolling tire with a single measurement instead of using the conventional experimental setup (i.e., measurement of the vibration of a rolling tire at multiple positions similar to the case of a non-rotating structure), which is used to estimate the dynamic behavior of a rolling tire. For this purpose HHT, which was used in the continuous measurement of a non-rotating structure in previous research studies, has been used for the case of a rotating system in this study. Ambiguous mode combinations can occur in this rotating system, and therefore, a method to overcome this ambiguity is proposed in this study. In addition, the specific phenomenon for a rotating system is introduced, and the effect of this phenomenon with regard to the obtained results through HHT is investigated.

\section{INTRODUCTION}

Research into the dynamic characteristics of rotating tires as well as on vehicle bodies has been actively carried out over the last decade because of the rapid increase in the number of vehicles ${ }^{1-4}$. Unlike a general mechanical system, a rotating tire has two different directional waves (forward and backward) with respect to the rotating direction. To verify these waves 
experimentally, mode-shape confirmation has to be accompanied. For mode-shape confirmation, measurements made at multiple points are required, but due to the nature of the rolling tire, special equipment is needed ${ }^{3}$. In this research study, a noncontact sensor, such as a Laser Doppler Vibrometer, is used to measure the vibration of a rolling tire. The Hilbert-Huang Transform (HHT) ${ }^{5}$ widely used in many fields (acoustics, radar and vibration signal analysis etc.) is applied to extract the mode-shape information under the assumption that the mode-shape information is included in a single-point measurement. Kang et al. ${ }^{6}$ continuously measured a beam that was excited by a single frequency while moving the laser beam at a constant speed from one end to the other end. The Operating Deflection Shape (ODS) is confirmed by applying the Hilbert Transform (HT) to the measurement. Recently, Kyong et al. ${ }^{7}$ introduced a method to extract mode shapes by applying HHT from a continuous measurement that is excited by an impact hammer. In this research study, the HHT is adapted because of the similarity of the experimental configuration in this study (fixed sensor, rotating structure) to a continuous measurement in previous research studies (fixed structure, moving sensor). In Ref. 6, the authors used a filter to apply the HHT, whereas, in this study, the mode shape is obtained through modal analysis by applying the HHT to the continuous measurement of an impulse response. This HHT with the modal approach has recently been introduced by Lee et al. ${ }^{8}$

In Section II, the HHT procedure to extract the mode shape in the previous research study ${ }^{7}$ is introduced in a simplified manner, and the phenomenon (Doppler Effect) that occurred when continuous measurement is conducted, is also introduced. In Section III, the theoretical background that is used for applying the HHT technique to a rolling tire is introduced. In Section A, the Frequency Response Function (FRF) of a rolling tire is induced through the equation of motion of a flexible ring model under the assumption that the system is modeled as a flexible ring. In Section B, the relationship between the induced FRF and HHT is investigated. In this section, the differences in the characteristics between the beam (used in the previous study) and the rolling tire (used in this study) are highlighted, and focus is placed on finding a way to overcome this discrepancy. In Section IV, experimental verification of the proposed method is performed. In Section C, mode-shape results are shown, which are obtained by the conventional modal parameter estimation method. These results are compared and analyzed with the results obtained from the proposed method. In Section D, 2 measurements are selected among several numbers of measurements, which were obtained in the previous section, and the HHT is applied to these selected measurements. Most importantly, the method introduced to overcome the differences highlighted in section B is explained in detail through these measurements, and the combination of ambiguous modes occurring in this process is also introduced. Furthermore, a detailed explanation of how to distinguish between the combination of ambiguous modes and the combination of actual modes is presented. In Section V, the conclusions drawn in this study are highlighted. 


\section{PROCEDURE TO RECONSTRUCT THE MODE SHAPE BY THE HHT}

The HHT has been applied in various fields, such as fluid dynamics, acoustics, and financial predictions, since Huang et al. ${ }^{5}$ introduced this method to evaluate linear and non-linear signals. Recently, Kyong et al. ${ }^{7}$ extended the application of the HHT to vibration measurements to reconstruct mode shapes. In this section, a detailed theoretical background of the HHT is not included, but a brief procedure to extract the mode shape is introduced with an example using a simply supported beam. In addition, the Doppler effect, which was not considered in the reference but should be considered for the continuous scanning measurement, will be explained.

$$
u\left(x_{s}, x_{f}, t\right)=\sum_{n=1}^{N} Y_{n}\left(t, \omega_{n}\right) \phi_{n}\left(x_{s}\right) \phi_{n}\left(x_{f}\right)
$$

where

$$
\begin{aligned}
& Y_{n}\left(t, \omega_{n}\right)=\frac{1}{M} e^{-\zeta_{n} \omega_{n} t} \sin \left(\omega_{d n} t+\theta_{n}\right), \omega_{d n}=\omega_{n} \sqrt{1-\zeta_{n}^{2}} \\
& \phi_{n}(x)=\sqrt{2} \sin \left(k_{n} x\right), \quad k_{n}=\frac{n \pi}{L}
\end{aligned}
$$

In Equation (1), $u$ represents the impulse response of a simply supported beam, $x$ indicates position in the $x$ direction, and subscripts $s$ and $f$ represent the measurement position and the forcing position, respectively. It has been confirmed that the impulse response function is a function of both time and space, which is determined by the measurement position and the forcing position. Furthermore, $Y$ represents the modal displacement, $\varphi$ represents the mode shape, $\omega_{n}$ represents the natural frequency, and the subscript $n$ represents the mode number. In Equation (1a), $M$ indicates the mass per unit length, $\zeta$ indicates the modal damping, $\omega_{d n}$ the resonance frequency, and $\Theta_{n}$ the phase difference. In Equation $(1 \mathrm{~b}), \mathrm{L}$ is the length of the beam. If the forcing position is chosen and the propagating vibration from the position is continuously measured from $x=0$ to $\mathrm{L}$ at a constant speed, then the measurement position $x_{\mathrm{s}}$ can be represented as

$$
x_{s}=L \Omega t
$$

Here, $\Omega$ is the frequency of the scanning speed. Equation (1) can be represented by substituting Equation (2) into it: 


$$
u(t)=\sum_{n=1}^{N} A_{n} e^{-\zeta_{n} \omega_{n} t} \sin \left(\omega_{d n} t+\theta_{n}\right) \sin (n \pi \Omega t) \quad \text { where } \quad A_{n}=\frac{1}{M} \sin \left(k_{n} x_{f}\right)
$$

That is, the impulse response function previously expressed as a function of time and space is represented here as a function of time only. In this equation, $A_{n}$ is a constant weighting factor of each mode, which is constituted by the mass of the beam and the mode shape in the forcing position. By using this equation, one can obtain the result shown in Figure 1 using $n=2, \omega_{n}=$ $220 \mathrm{~Hz}$ and $\mathrm{L}=0.45 \mathrm{~m}$ for the undamped case, while measuring the structure with $\Omega=13 \mathrm{~Hz}$.

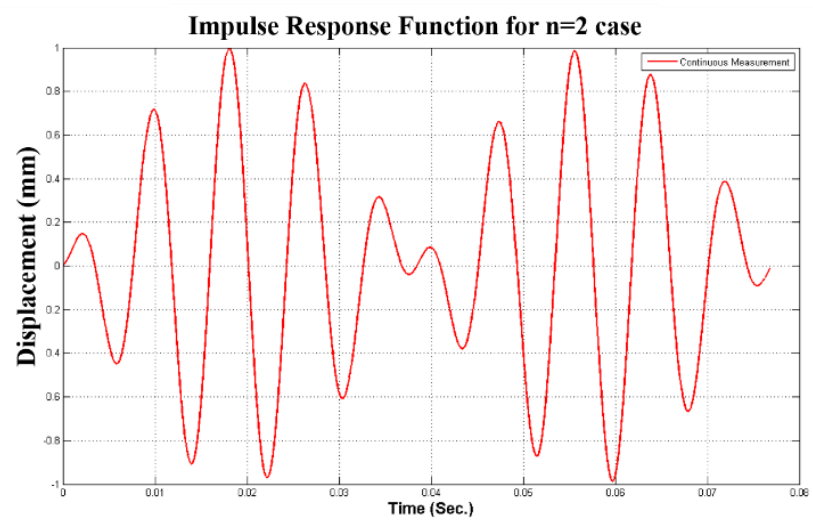

FIG. 1. Continuous scanning measurement of 2nd mode for the undamped impulse response.

The result shown in Figure 1 can be applied to the HHT to extract the mode shape of the $2^{\text {nd }}$ mode. This procedure is described below.
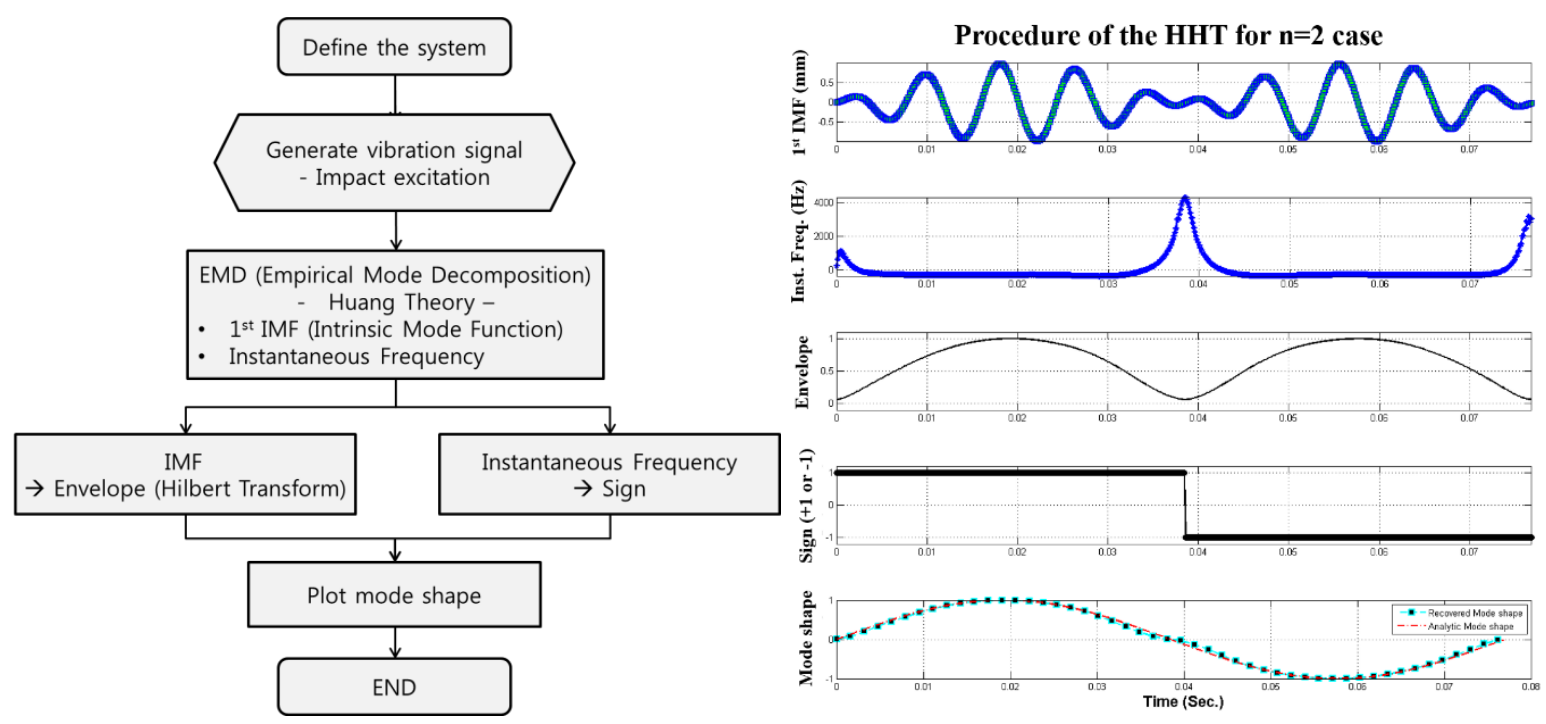
FIG. 2. (a) A schematic diagram of the HHT (b) from top to bottom: the $1^{\text {st }}$ intrinsic mode function of impulse response of the $2^{\text {nd }}$ mode, the instantaneous frequency, the normalized envelope, the sign obtained from the instantaneous frequency, the reconstructed mode shape (- - -) and the analytic mode shape $(-\cdot-)$.

Figure 2 (a) shows the schematic diagram of the process to extract the mode shape using the HHT. First, as obtained in Figure 1, the undamped impulse response is applied to the Huang theory. One can obtain a $1^{\text {st }}$ intrinsic mode function (IMF) using an empirical mode decomposition (EMD) process as the first basis in time. The IMF is used to obtain the envelope using the Hilbert transform, and the peak position of the instantaneous frequency is used to determine the sign of the envelope. The mode shape can be obtained by using the envelope and the sign. Figure 2 (b) shows the results obtained in each step depicted in Figure 2 (a) using the results from Figure 1. From top to bottom, the first plot shows the $1^{\text {st }}$ IMF of the result obtained from Figure 1 by applying the EMD process, and the second plot shows the instantaneous frequency, which is also a result obtained from the EMD process. The third plot shows the envelope obtained by applying the Hilbert transform to the $1^{\text {st }}$ IMF with the magnitude normalized. The fourth plot shows the sign determined by the instantaneous frequency. Here, one can observe that the change in sign occurs at the location of the maximum peak value of the instantaneous frequency. This sign can be changed depending on how the first value has been selected. For instance, if one assigns -1 as a starting value, the sign should change to +1 at the peak location of the instantaneous frequency, but the final reconstructed mode shape would remain the same whether +1 or -1 is used as the starting value. Moreover, the physical meaning of the peak value in the instantaneous frequency indicates the nodal position of the vibration, and one can expect an abrupt change of the instantaneous frequency as the sensor passes through this position.

Finally, the fifth plot shows two mode results. One is the reconstructed mode shape obtained by combining the envelope and the sign, and the second is the analytical mode shape from Equation (1b). As shown in the plot, the reconstructed result from the HHT strongly agrees with the analytical result. Figure 2 shows the overall process used in the reference $e^{4}$ to obtain the mode shape. In the reference, however, two additional techniques (the ideal band pass filter and compensation damping) were implemented to obtain the un-damped impulse response to the continuous scanning measurement of an impact test, but in the present study, only the modal analysis method has been used to obtain an accurate result. A detailed explanation is given in the next section. Next, we tried to identify the inherent features of the continuous scanning measurement of an impact test. Equation 
(3) represents the response of an impact through a continuous scanning measurement, which can be re-represented by applying trigonometric identities.

$$
u(t)=\sum_{n=1}^{N} \frac{1}{2} A_{n} e^{-\zeta_{n} \omega_{n} t}\left[\begin{array}{l}
\cos \theta_{n}\left\{\sin \left(\left(\omega_{d n}+n \pi \Omega\right) t\right)-\sin \left(\left(\omega_{d n}-n \pi \Omega\right) t\right)\right\} \\
-\sin \theta_{n}\left\{\cos \left(\left(\omega_{d n}+n \pi \Omega\right) t\right)-\cos \left(\left(\omega_{d n}-n \pi \Omega\right) t\right)\right\}
\end{array}\right]
$$

From Equation (4), one can see that the resonance frequency in non-continuous (discrete) measurements has been shifted towards the positive and the negative directions in a continuous scanning measurement by an amount equal to $n \pi \Omega$. This fact indicates that the resonant frequency in a non-continuous measurement is shown by a peak at a single position in the frequency domain, while in continuous scanning measurements, two peaks are shown at positions that are shifted by an amount $\pm n \pi \Omega$ relative to the original resonance frequency. This phenomenon is called the Doppler effect. Although this effect was not considered in the reference study, it is an important consideration in the present study (vide infra). In addition, this equation confirms that the two frequency components are combined with the same phase $\left(\theta_{n}\right)$; therefore, the beating phenomenon can occur for two frequencies near the resonance frequency of the structure. The envelope frequency of this beating phenomenon can be represented as follows: ${ }^{9}$

$$
f_{m}=\frac{1}{2}\left\{\left(\omega_{d n}+n \pi \Omega\right)-\left(\omega_{d n}-n \pi \Omega\right)\right\}=n \pi \Omega
$$

It is confirmed from Equation (5) that the envelope frequency from the beating phenomenon is represented by the combination of the mode number and the scanning frequency only. Thus, $n$ envelope variations would be expected during one scanning revolution (from one end to the other end of the beam $0 \sim \mathrm{L}$ ). Finally, the HHT is only a technical method to extract mode information $(n)$ from the continuous scanning measurement of an undamped impulse response..

\section{FLEXIBLE ROTATING RING}

In this section, it is assumed that a rotating tire can be analyzed by a flexible-ring model as in previous papers ${ }^{1,10-11}$. The impulse response for this model is investigated in the time domain (Impulse Response Function) and in the frequency domain (Frequency Response Function). The method to estimate mode shape by applying the HHT to these induced numerical models will be investigated in this paper. 


\section{A. Frequency Response Function for a rotating flexible-ring model in fixed reference frame}

A ring is represented by cylindrical coordinates, and two coordinates can be used to analyze the dynamic behavior. One is a local coordinate (co-rotating coordinate) and another one is a global coordinate (fixed reference) ${ }^{10-11}$.

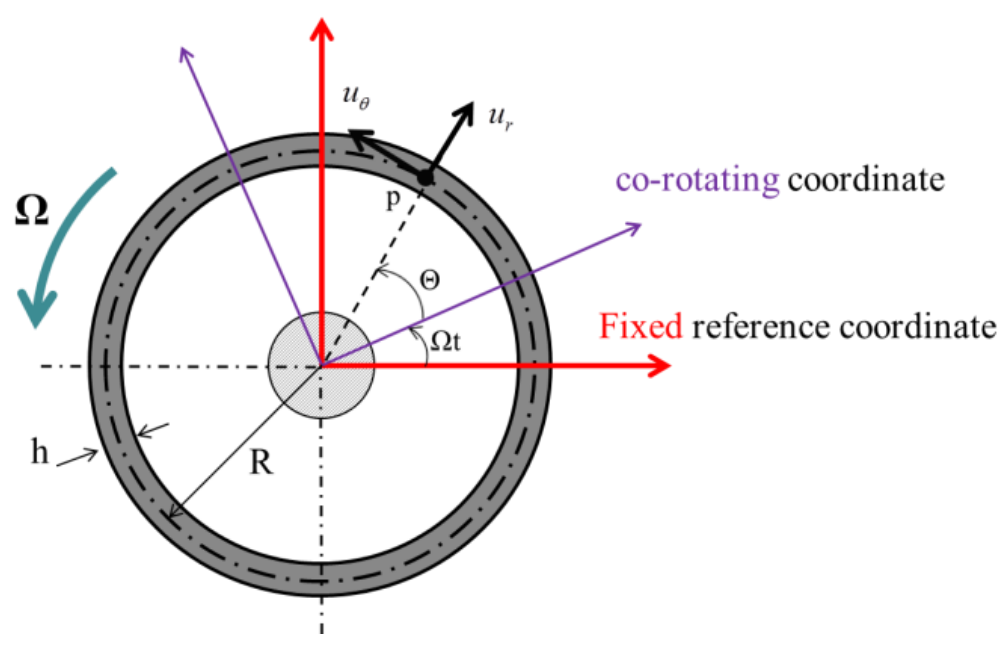

FIG. 3. Global and local coordinates of a rotating flexible ring.

Figure 3 shows the configuration of the rotating flexible ring and its two coordinate systems (global and local); if a rotating tire is measured using a contact sensor such as an accelerometer, the vibrational behavior can be represented by a local coordinate, whereas if a non-contact sensor, such as a laser Doppler vibrometer, is adopted for obtaining the vibrational information of the tire, then the global coordinate can be adopted. The equation of motion of a rotating ring considering damping in local coordinates is as follows ${ }^{10-11}$.

$$
\mathbf{M} \ddot{\mathbf{u}}(\theta, t)+\mathbf{C} \dot{\mathbf{u}}(\theta, t)+\mathbf{K u}(\theta, t)=\mathbf{q}(\theta, t)
$$

where,

$$
\begin{gathered}
\mathbf{u}=\left\{\begin{array}{l}
u_{r} \\
u_{\theta}
\end{array}\right\}, \mathbf{q}=\left\{\begin{array}{c}
q_{r} \\
q_{\theta}
\end{array}\right\} \\
u_{r}(\theta, t)=U_{r} e^{j(n \theta+\omega t)}, u_{\theta}(\theta, t)=U_{\theta} e^{j(n \theta+\omega t)} \\
q_{r}(t)=Q_{r} e^{j \omega t}, q_{\theta}(\theta, t)=Q_{\theta} e^{j \omega t}
\end{gathered}
$$


In Equation (6), the $\mathbf{M}, \mathbf{C}$ and $\mathbf{K}$ matrices represent the mass, gyroscopic and stiffness matrices, respectively, and the detail configuration of these matrices is introduced in Refs. 10-11. The vectors $\mathbf{u}$ and $\mathbf{q}$ represent displacements and external forces in the radial and circumferential directions, respectively. The size of each matrix is $2 \mathrm{~N} \times 2 \mathrm{~N}$, and the detailed description of these matrices and vectors are shown in Eq. (6a) and (6b), respectively. As shown in the reference paper, the mass matrix is diagonal and composed of real values, the gyroscopic matrix is skew-symmetric where it is also composed of real values, and the stiffness matrices are skew-symmetric matrices where they are composed of complex values. To obtain the natural frequency of the system using Equation (6), the eigenvalue problem should be solved, but it is impossible to solve the general eigenvalue problem due to the gyroscopic matrix. Therefore, this equation should be transformed into state space: ${ }^{11-12}$

$$
\mathbf{A} \dot{\mathbf{p}}+\mathbf{B} \mathbf{p}=\mathbf{f}
$$

where,

$$
\mathbf{A}=\left[\begin{array}{cc}
\mathbf{0} & \mathbf{M} \\
\mathbf{M} & \mathbf{C}
\end{array}\right], \mathbf{B}=\left[\begin{array}{cc}
-\mathbf{M} & \mathbf{0} \\
\mathbf{0} & \mathbf{K}
\end{array}\right], \mathbf{p}=\left\{\begin{array}{l}
\dot{\mathbf{u}} \\
\mathbf{u}
\end{array}\right\} \text { and } \mathbf{f}=\left\{\begin{array}{l}
\mathbf{0} \\
\mathbf{q}
\end{array}\right\}
$$

Matrices A and B in Equation (7) include imaginary values and are not symmetric matrices. If one solves the eigenvalue problem without external force (i.e., $\mathrm{f}=0$ ), then one can obtain eigenvalue results in the radial direction as shown below.

$$
\lambda_{n F}=-\sigma_{n F}-j \omega_{n F}, \lambda_{n B}=-\sigma_{n B}+j \omega_{n B}
$$

In Equation (8), subscript $\mathrm{n}$ represents the mode number. F and B represent the forward and backward modes, respectively, which means that every mode has two eigenvalues in which one is for the forward propagating wave and another one is for the backward propagating wave. These two waves occurred by the bifurcation effect due to the gyroscopic matrix in Equation (6). Each eigenvalue is composed of a real and an imaginary value where the real part stands for the energy dissipation and the imaginary part stands for the resonance frequency. Here, it should be noted that the resonance frequencies for forward and backward waves are represented by negative and positive quantities, respectively. In this study, the laser Doppler vibrometer is used to measure the vibration of a rotating tire, and therefore, the dynamic behavior should be analyzed in a fixed reference frame. Equation (6) represented in a local coordinate should be transformed into a global coordinate. This coordinate transformation can be performed by means of Reynolds' theorem ${ }^{11,13}$, 


$$
\frac{D}{D t}=\frac{\partial}{\partial t}+\Omega \frac{\partial}{\partial \theta}
$$

The left hand side of Eq. (9) represents the local coordinate (Lagrangian), and the first term on the right hand side represents the global coordinate (Eulerian). The Greek letter $\Omega$ represents the rotational speed of the ring. If one applies this theorem, which is represented by the total derivative of Equation (6) that is represented by a partial derivative, then the resultant matrices are represented by the same mass, gyroscopic and stiffness matrices as Equation (6). The components of the matrices in global coordinates have been changed, and the gyroscopic matrix, which was only composed of real values, now also includes imaginary values ${ }^{11}$. If one solves the eigenvalue problem with matrices in global coordinates, one can obtain the following results,

$$
\lambda_{n F}=-\sigma_{n F}-j\left(\omega_{n F}+2 n \pi \Omega\right), \lambda_{n B}=-\sigma_{n B}+j\left(\omega_{n B}-2 n \pi \Omega\right)
$$

Equation (10) shows the eigenvalues of a flexible-ring model in global coordinates that have different values for each directional wave, which is the same as Equation (8). The difference between Equation (8) and Equation (10) is that the imaginary results of Equation (10) are shifted to the negative direction by an amount $n \Omega$ compared to Equation (8) by the Doppler effect, as shown in the last section with the static example. This effect, which occurs in global coordinates, has been reported in several previous research studies ${ }^{11-13}$. Because the eigenvalues obtained in global coordinates have been explained previously, the eigenvectors will be considered. These vectors are obtained in the state space, and the obtained eigenvectors and adjoint-eigenvectors satisfy the bi-orthogonal properties with the system matrices $\mathbf{A}$ and $\mathbf{B}$. From these properties ${ }^{11}$, the radial directional FRF can be represented by the following.

$$
H(\theta, j \omega)=\sum_{n=1}^{N} \sum_{i=F, B}\left\{\frac{B \varphi_{\mathbf{n}} \psi_{\mathbf{n}}^{\mathbf{H}}}{\left(j \omega-\lambda_{n}\right)}+\frac{\bar{B} \bar{\varphi}_{\mathbf{n}} \psi_{\mathbf{n}}^{\mathbf{T}}}{\left(j \omega-\bar{\lambda}_{n}\right)}\right\}^{i}
$$

$$
\text { where } B=e^{j n\left(\theta_{s}-\theta_{f}\right)}
$$

Equation (11) shows the frequency response function of a flexible-ring model in global coordinates. Here, superscripts $\mathrm{H}$ and $\mathrm{T}$ represent the Hermitian matrix and transposed matrix, respectively. The modal vector and adjoint-modal vector are

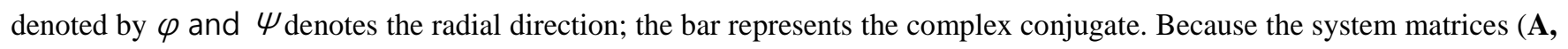


B) are non-symmetric, the obtained modal and adjoint-modal vectors have different values in the different directional components (i.e., radial and circumferential direction). The Maxwell's reciprocity theorem, which holds for a static system, does not hold for the rotating condition because of this difference. In addition, the constant B, the value of which is decided by observing the position (subscript s) and forcing position (subscript f), represents the phase information of each mode. If the forcing and observing positions are switched, then this phase has a different sign. This result is further proof of the fact that the theorem does not hold for the rotating condition.

\section{B. Relationship between FRF and HHT}

In this section, the relationship between the induced FRF and HHT will be investigated. First, Equation (11) can be rerepresented as follow.

$$
H(\theta, j \omega)=\sum_{n=1}^{N} \sum_{i=F, B}\left\{\frac{R_{n} e^{j \theta_{n}}}{\left(j \omega-\lambda_{n}\right)}+\frac{R_{n} e^{-j \theta_{n}}}{\left(j \omega-\bar{\lambda}_{n}\right)}\right\}^{i}
$$

Equation (12) represents the numerator part of Equation (11) with phase and magnitude information. If this equation, which is represented in frequency domain, is converted into the time domain by an inverse Laplace transform, one obtains:

$$
h(t)=\sum_{n=1}^{N} \sum_{i=F, B}^{i}\left\{R_{n} e^{-\sigma_{n} t}\left(e^{j\left(\omega_{n} t+\theta_{n}\right)}+e^{-j\left(\omega_{n} t+\theta_{n}\right)}\right)\right\}=\sum_{n=1}^{N} \sum_{i=F, B}^{i}\left\{2 R_{n} e^{-\sigma_{n} t} \cos \left(\omega_{n} t+\theta_{n}\right)\right\}
$$

Equation (13) represents the impulse response by a mode summation approach in the time domain. The left part of this equation is obtained from the inverse Laplace transform of Equation (12), and the right part shows the left part by applying Euler's equation to the same. So far, Equations (11) - (13) represented by the mode summation method separate the forward and backward waves for each mode, but Equation (14) is represented without separating the forward and backward waves.

$$
h(t)=\sum_{n=1}^{N} 2 R_{n}\left\{e^{-\sigma_{n F} t} \cos \left(-\left(\omega_{n F}+2 n \pi \Omega\right) t+\theta_{n}\right)+e^{-\sigma_{n B} t} \cos \left(\left(\omega_{n B}-2 n \pi \Omega\right) t+\theta_{n}\right)\right\}
$$

The damping and resonance frequency in eq. (14) are represented in Equation (10). Modal parameters (natural frequency, damping and modal participation factor) in this equation can be estimated by applying EMA). These estimated parameters from experimental measurements can be used to obtain the impulse response by substituting the estimated parameters into eq. (14). As shown in section II, the HHT can only be used in the case of an undamped response. Therefore, in the process of obtaining 
the impulse response through Equation (14), it is compulsory to assign a value of 0 to the damping part; then, the undamped impulse response of the continuous measurement can be obtained by modal parameter estimation. Trigonometric identities can be applied to this result with the assumption that the magnitude of each phase of different directivity waves are similar to each other at low rotating speed (i.e., $\left|\theta_{n F}\right| \approx\left|\theta_{n B}\right|$ ). Its resultant is shown as follows:

$$
h(t)=\sum_{n=1}^{N} 2 R_{n}\left\{\begin{array}{l}
\cos \left(\omega_{n F}+2 n \pi \Omega\right) t \cos \left(\theta_{n}\right)+\sin \left(\omega_{n F}+2 n \pi \Omega\right) t \sin \left(\theta_{n}\right) \\
+\cos \left(\omega_{n B}-2 n \pi \Omega\right) t \cos \left(\theta_{n}\right)-\sin \left(\omega_{n B}-2 n \pi \Omega\right) t \sin \left(\theta_{n}\right)
\end{array}\right\}
$$

From eq. (15), it is confirmed that the vibration by the resonance frequency is altered by the weighting factor (sine and cosine), which has the same phase angle. It can be rearranged as follows:

$$
h(t)=\sum_{n=1}^{N} 2 R_{n}\left[\begin{array}{l}
\cos \left(\theta_{n}\right)\left\{\cos \left(\omega_{n F}+2 n \pi \Omega\right) t+\cos \left(\omega_{n B}-2 n \pi \Omega\right) t\right\} \\
+\sin \left(\theta_{n}\right)\left\{\sin \left(\omega_{n F}+2 n \pi \Omega\right) t-\sin \left(\omega_{n B}-2 n \pi \Omega\right) t\right\}
\end{array}\right]
$$

Equation (16) is similar to Equation (4), and therefore, it can be estimated that the combination of the forward and backward waves for the undamped impulse response will construct an envelope at the resonance frequency.

$$
f_{m}=\frac{1}{2}\left\{\left(\omega_{n F}+2 n \pi \Omega\right)-\left(\omega_{n B}-2 n \pi \Omega\right)\right\} \approx 2 n \pi \Omega
$$

Equation (17) represents the frequency of the resultant envelope for an undamped impulse response. If the rotating speed (rotating frequency) of the ring is less than the frequency of each mode, then the Coriolis effect will be small (i.e., $\omega_{n F} \approx \omega_{n B}$ ); in this case, the frequency of the envelope can be represented by only mode information. This information can be extracted by the HHT. It should be noted that the important thing is to find the forward and backward waves for each mode. In the EMA of a rotating tire, the force is transmitted from a cleat, and this force has similar but not the same contents as an impulse in the frequency domain. It is confirmed that there is no energy in a certain frequency region when this force is analyzed in the frequency domain ${ }^{14-15}$. This finding means that sufficiently excited modes can be observed but insufficiently excited modes cannot be observed. Therefore, some modes are observed as a pair (forward and backward), while other modes are not (i.e., only one of two waves is observed). In addition, for the rotating tire case, several modes are clustered in the interesting frequency region $(0 \sim 500 \mathrm{~Hz})$, unlike the beam case in the reference ${ }^{7}$, in which each mode is well separated. As a result, it is 
important to find waves that constitute a pair in which each wave has a different direction. For this purpose, the phase of each directional wave will be used. The weighting factor, as represented by the cosine and sine in Equations (15) and (16), is represented as a numerator in Equation (12), and this will be represented as a real- and imaginary-part of residue when it is represented by a complex value, which can be estimated by the modal analysis method. In addition, the weighting factor in the cosine case is represented by the same sign for the forward and backward waves, but the weighting factor in the sine case is represented by a different sign. Therefore, when every residue estimated by EMA for each mode is represented in the Cartesian coordinate frame (X [real part] - Y [imaginary part]), it can be expected that each pair is symmetric about the X-axis. This fact can be used to find out pair waves. However, when every pair of each mode estimated by experiment is plotted in Cartesian coordinates, it will be fortunate if the results are exactly symmetric about the X-axis. In that case, it would be difficult for the case in which several modes are located in a certain region as a cluster in Cartesian coordinates, and then, it can be ambiguous to identify whether it is symmetric or not. For this case, two waves that are anticipated to make up a pair can be selected, and if one constitutes an undamped impulse response using Equations (15) and (16), then, the numerical configuration of this response is as follows:

$$
h_{12}(t)=\left[\begin{array}{l}
\left\{R_{1} \cos \left(\theta_{1}\right) \cos \left(\omega_{1} t\right)+R_{2} \cos \left(\theta_{2}\right) \cos \left(\omega_{2} t\right)\right\} \\
+\left\{R_{1} \sin \left(\theta_{1}\right) \sin \left(\omega_{1} t\right)+R_{2} \sin \left(\theta_{2}\right) \sin \left(\omega_{2} t\right)\right\}
\end{array}\right]
$$

The subscripts 1 and 2 in Equation (18) represent any two selected modes. If these two modes (anticipated as a pair) do not make up a pair as forward and backward in one mode number, then these two waves are amplified by different magnitudes and different phases, respectively. Eventually, it can be estimated that the envelope and instantaneous frequency obtained via the HHT show different configurations against what we expected.

\section{EXPERIMENT}

In this section, the experimental configuration and the validation of the proposed method and its results are discussed. First, the vibration of a rolling tire is measured at 45 nodes. Then, the experimental configuration is introduced, and finally the obtained results (natural frequency, damping and mode shape) are presented in section C. In section D, two measurements among the 45 nodes are selected, to which are applied the HHT, and the obtained results from these two measurements are 
verified. In addition, these results are compared with the results obtained in section C. Moreover, discussion and comparison of the HHT are included.

\section{Experimental configuration}

We have developed an experimental setup for a rotating tire in this study.

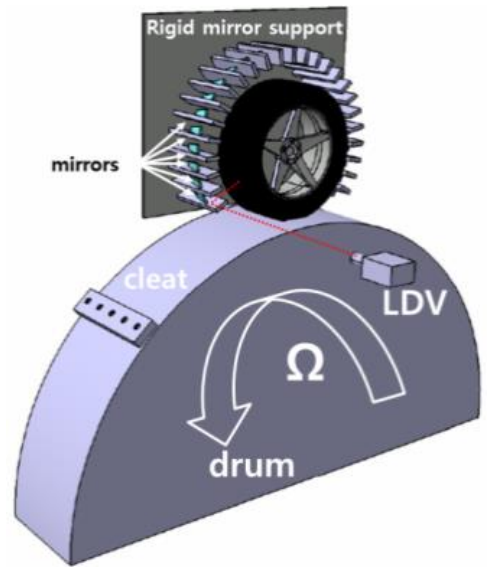

FIG. 4. Configuration for the vibration measurement of a rolling tire.

As shown in Figure 4, the tire comes in contact with a large drum during rotation. A cleat is attached to the drum. This cleat is used to apply a force similar to an impulse for every revolution of the drum. The vibration generated by the applied force is measured using a laser Doppler vibrometer (Polytec OFV-353 sensor head with OFV-2200-H controller). For convenient measurement, a fixed frame composed of 45 mirrors is installed on the back side of the tire. These mirrors are evenly distributed every $6^{\circ}$ starting from $315^{\circ}$ to $225^{\circ}$ in the rotating direction of the tire with respect to the contact surface $\left(270^{\circ}\right)$ (refer to Figure 4). The mirrors are installed to direct the laser beam to the center of the rotating tire cross-section. The experiment is conducted for the $100 \mathrm{~km} / \mathrm{h}$ case, and the reading is taken for 10 seconds at each mirror. In addition, the tire is fixed to the hub at its center (i.e., the rotational axis of the tire). A force transducer is attached to the hub, and this sensor measures the transmitted force in the z-direction (normal to the hub). This reading always shows an equivalent measurement for each node, even though the laser sensor is directed at a different position. In addition, the first resonance frequency of the hub in z-direction is larger than the frequencies of interest $(0-500 \mathrm{~Hz})$. Therefore, the dynamic characteristics of the hub do not affect the measurements in this region. This force measurement at each node is used as a trigger to find the average measurement for a 10-second duration and is also used as a reference in EMA, which means that this signal constitutes cross correlation functions with 45 measurements. 
The measurement conditions are listed in Table I. A more detailed explanation of the experimental setup can be found in Ref. 11.

TABLE I. Measurement conditions for the rolling tire

\begin{tabular}{lc}
\hline \multicolumn{2}{c}{ Measurement conditions } \\
\hline \hline Tire rolling speed $[\mathrm{km} / \mathrm{h}]$ & 100 \\
Tire dimensions & $205 / 55 \mathrm{R} 16$ \\
Inflation Pressure [bar] & 2.2 \\
Temperature & $50^{\circ} \mathrm{C}$ \\
Load [N] & 4000 \\
Applied Excitation & $3 \times 25 \mathrm{~mm}$ cleat \\
Sampling Frequency $[\mathrm{kHz}]$ & 64 \\
\hline \hline
\end{tabular}

In this study, PolyMAX is adopted to identify the dynamic characteristics of a rolling tire ${ }^{16}$. Figure 5 shows the average power spectra (solid line) at every node and reconstructed power spectra (solid and dotted line) using PolyMAX. As shown in the figure, it is confirmed that these two lines show good agreement in the frequency region of interest. Therefore, it is reliable for the obtained modal parameters (natural frequency, damping and mode shape). All of the estimated results of the modal parameters from 45 measurements are listed in Figure 6.

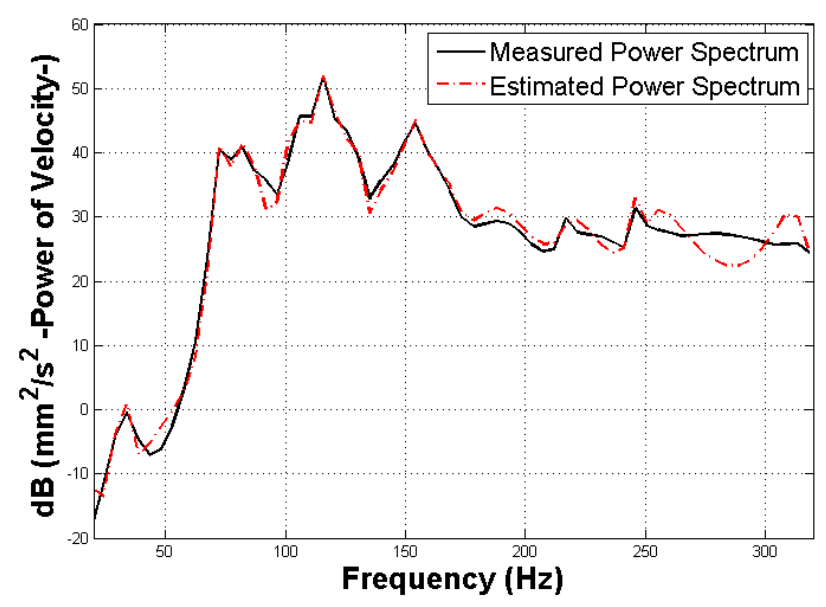

FIG. 5. Measurement (solid line) and estimated results (dotted line); response power spectrum and its estimation for a rolling tire (100 km/h). 


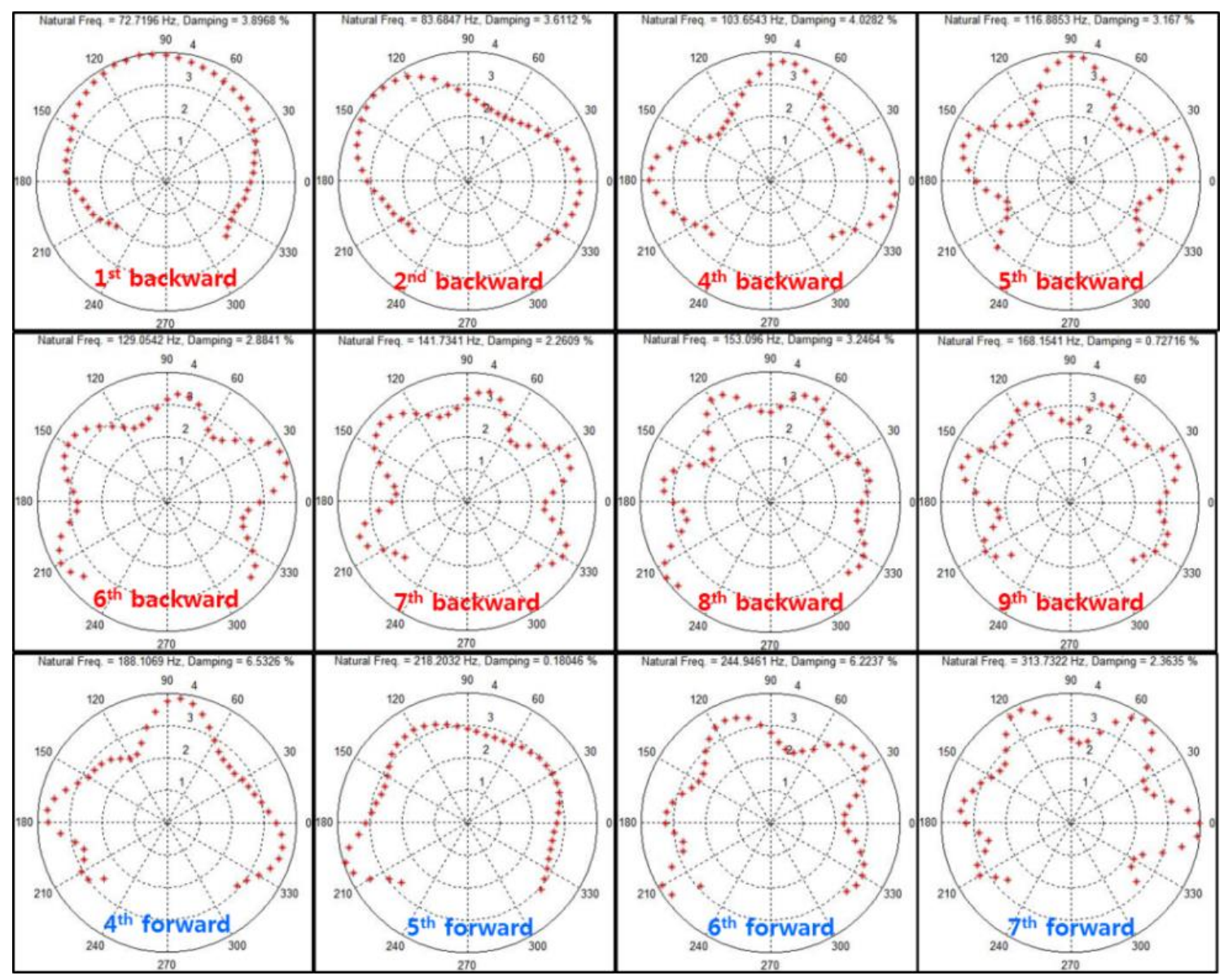

FIG. 6. Mode shape for the rolling tire at $100 \mathrm{~km} / \mathrm{h}$.

In Figure 6, the terms backward and forward represent the wave directivity observed from the mode shape. It is confirmed that some of the modes are not observed in the figure because the frequency contents of the cleat-applied force are dependent on the rotating speed and geometrical configuration of the cleat ${ }^{14-15}$. It shows that the higher the speed, the greater the number of modes that can be observed. The estimated results in Figure 6 are listed in Table II.

TABLE II. Modal parameter estimation results from 45 measurements 


\begin{tabular}{|c|c|c|}
\hline \multicolumn{3}{|c|}{$100 \mathrm{Km} / \mathrm{h}$} \\
\hline \multirow[b]{2}{*}{$\begin{array}{c}\text { Mode } \\
\text { Number }\end{array}$} & \multirow[b]{2}{*}{$\begin{array}{l}\text { Wave } \\
\text { direction }\end{array}$} & $\begin{array}{c}\text { PolyMAX } \\
\text { (with } 45 \text { measurments) }\end{array}$ \\
\hline & & Freq. $(\mathrm{Hz}) /$ Damping (\%) \\
\hline \multirow{2}{*}{1} & $1 B$ & $72.72 / 3.9$ \\
\hline & $1 \mathrm{~F}$ & * \\
\hline \multirow{2}{*}{2} & $2 \mathrm{~B}$ & 83.69 / 3.61 \\
\hline & $2 \mathrm{~F}$ & * \\
\hline \multirow{2}{*}{3} & $3 B$ & * \\
\hline & $3 F$ & * \\
\hline \multirow{2}{*}{4} & $4 B$ & $103.6 / 4.02$ \\
\hline & $4 \mathrm{~F}$ & $188.1 / 6.53$ \\
\hline \multirow{2}{*}{5} & $5 B$ & 116.9 / 3.17 \\
\hline & $5 F$ & $218.2 / 0.18$ \\
\hline \multirow{2}{*}{6} & $6 \mathrm{~B}$ & $129 / 2.88$ \\
\hline & $6 \mathrm{~F}$ & $244.95 / 6.22$ \\
\hline \multirow{2}{*}{7} & $7 B$ & $141.73 / 2.26$ \\
\hline & $7 F$ & $313.73 / 2.365$ \\
\hline \multirow{2}{*}{8} & $8 B$ & $153.1 / 3.25$ \\
\hline & $8 \mathrm{~F}$ & * \\
\hline \multirow{2}{*}{9} & $9 B$ & $168.1 / 0.72$ \\
\hline & $9 \mathrm{~F}$ & * \\
\hline
\end{tabular}

* indicates a non-identified mode

As discussed previously, every mode in the frequency region of interest is not identified by the cleat excitation, which is verified by Table II. Only four modes (mode number 4-7) are observed to have two directional waves, and hence, it is possible to extract the mode shape by applying the HHT for only these 4 modes. This is the estimation from 45 measurements and not from a single measurement. Consequently, to obtain reliable results from the HHT with a single measurement, identification of the number of pairs in the measurement should be known. To this end, the phase (weighting factor) that was introduced in section III will be investigated in this section.

\section{Applied results of the HHT}

Two arbitrary nodes $\left(20^{\text {th }}-72^{\circ}-, 2^{\text {th }}-126^{\circ}-\right)$ are selected from 45 nodes to validate the proposed HHT technique. First, every estimated weighting factor, which is represented in residues as a real and an imaginary part, is plotted in the $\mathrm{X}$-Y plane. 


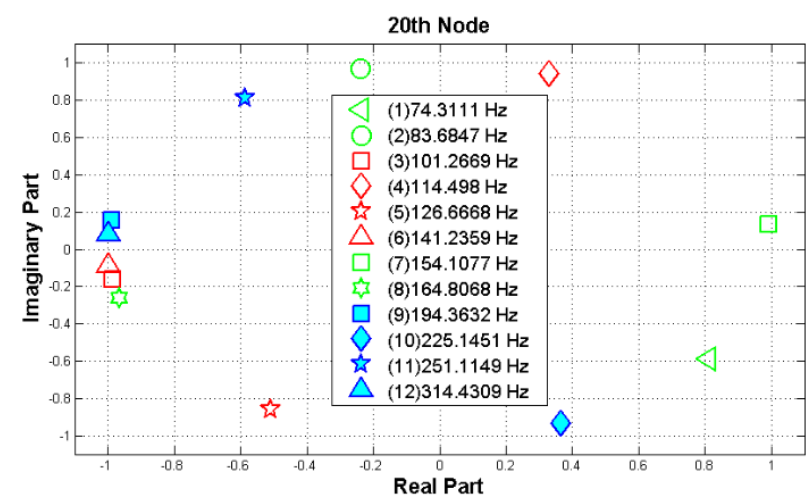

(a)

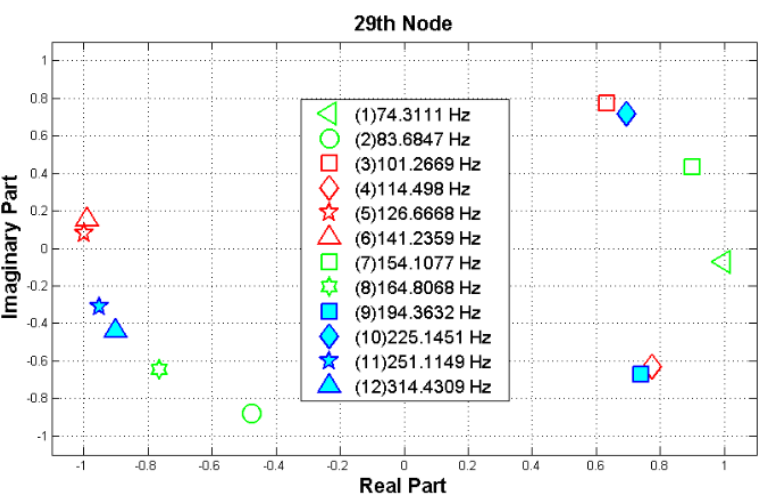

(b)

FIG. 7. Weighting factors plot for the estimated modes; $74 \mathrm{~Hz}(\triangleleft), 83 \mathrm{~Hz}(\circ), 101 \mathrm{~Hz}(\square), 114 \mathrm{~Hz}(\diamond), 126 \mathrm{~Hz}(\succsim), 141 \mathrm{~Hz}(\Delta), 154 \mathrm{~Hz}($

$\nabla), 164 \mathrm{~Hz}(\Varangle), 194 \mathrm{~Hz}(\boldsymbol{\bullet}), 225 \mathrm{~Hz}(\diamond), 251 \mathrm{~Hz}(\star), 314 \mathrm{~Hz}(\boldsymbol{\Delta})$ (a) weighting factors of the estimated modes at the 20th node, (b) weighting factors of the estimated modes at the $29^{\text {th }}$ node.

In Figure 7, the $X$ axis indicates the real part $(\cos (\theta))$ of the residue and the $Y$ axis indicates the imaginary part $(\sin (\theta))$ of the residue. Figure 7 shows the result of the $20^{\text {th }}$ and $29^{\text {th }}$ node. Here, the results in this figure show a small difference compared to Table II because the results in Table II are obtained from 45 measurements while the results in this figure are obtained from 2 measurements. The pair waves in the same mode are selected based on Table II, and each wave composing each pair is represented as a hollow shape for the backward wave and as a solid shape for the forward wave, respectively (e.g., $\diamond, \diamond$ or $\downarrow$ , $\star$ ). The results in Figure 7 are similar to those predicted by the case in which the pairs are symmetric about the $X$-axis. Some modes, however, look ambiguous, making it difficult to judge whether they would make a pair or not. For these cases, the envelope and instantaneous frequency are investigated by applying the HHT. The envelope and instantaneous frequency from the combination of these ambiguous modes are distinguished from the results of pair waves as described in the following 3 cases.

Case (1) distortion of the envelope

Case (2) distortion of the instantaneous frequency (having maximum values at an unexpected location or zigzag configuration)

Case (3) wrong wave number in the envelope at combining frequencies (i.e., the expected mode number at the combined frequencies is different from the estimated mode number in the envelope) 
To confirm, several combinations of waves that can be assumed to be ambiguous modes are investigated. From Figure 7 (a); these combinations are as follows: combination of $101 \mathrm{~Hz}(\square)$ and $314 \mathrm{~Hz}(\boldsymbol{\Lambda})$ (the HHT results of this combination are shown in Figure 8 (a)), combination of $164 \mathrm{~Hz}(\bowtie)$ and $194 \mathrm{~Hz}(\mathbf{})$ (the HHT results of this combination are shown in Figure 8 (b)), combination of $74 \mathrm{~Hz}(\triangleleft)$ and $114 \mathrm{~Hz}(\diamond)$ (the HHT results of this combination are shown in Figure 8 (c)) and combination of $154 \mathrm{~Hz}(\nabla)$ and $225 \mathrm{~Hz}(\bullet)$ (the HHT results of this combination are shown in Figure 8 (d)). And from Figure 7 (b); combination of $126 \mathrm{~Hz}($ 湤) and $314 \mathrm{~Hz}(\boldsymbol{\Delta})$ (the HHT results of this combination are shown in Figure 9 (a)), combination of $141 \mathrm{~Hz}(\Delta)$ and $164 \mathrm{~Hz}(\Varangle)$ (the HHT results of this combination are shown in Figure 9 (b)), combination of $74 \mathrm{~Hz}(\triangleleft)$ and 154

$\mathrm{Hz}(\nabla)$ (the HHT results of this combination are shown in Figure $9(\mathrm{c})$ ) and combination of $114 \mathrm{~Hz}(\diamond)$ and $154 \mathrm{~Hz}(\nabla)$ (the HHT results of this combination are shown in Figure 9 (d)). Each of these eight pairs is selected as an ambiguous wave combination. The envelope and instantaneous frequency in Figures 8 and 9 are obtained from these pairs by applying the HHT to an undamped impulse response obtained using Equation (18).

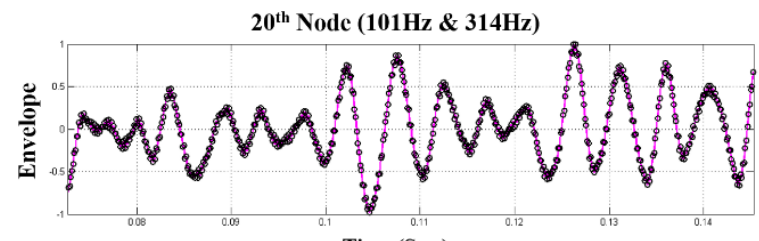

Time (Sec.)

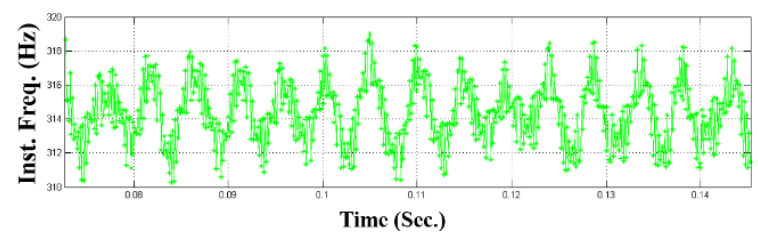

(a)

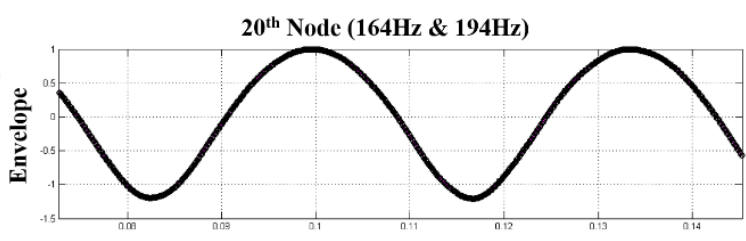

Time (Sec.)

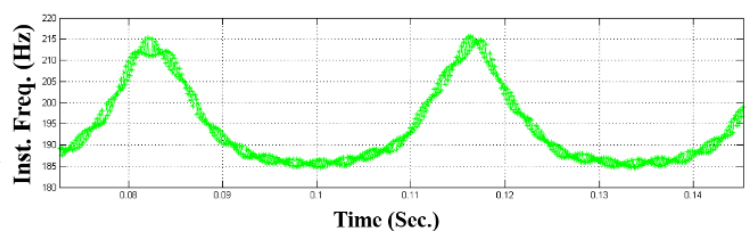

(b) 

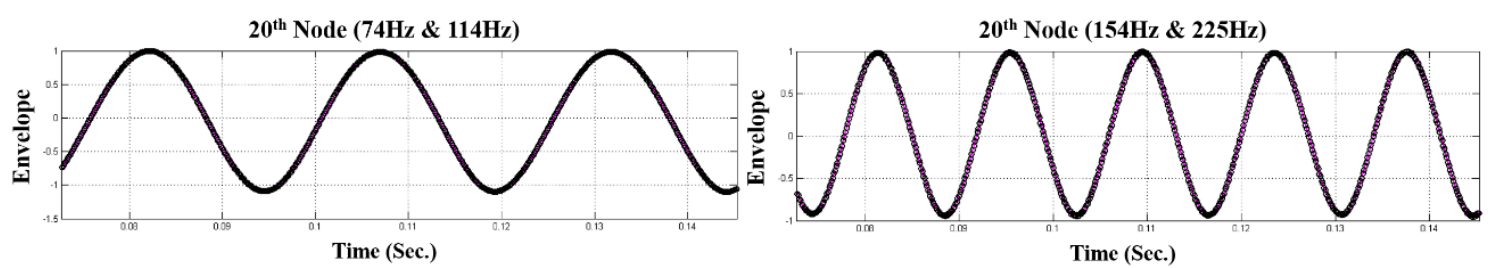

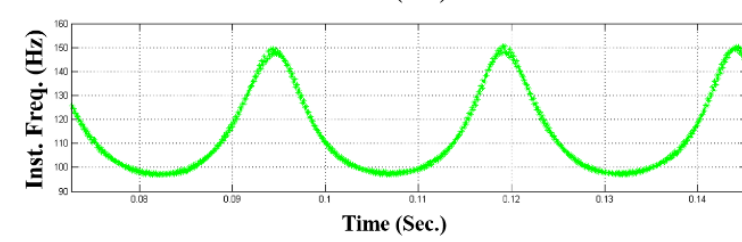

(c)

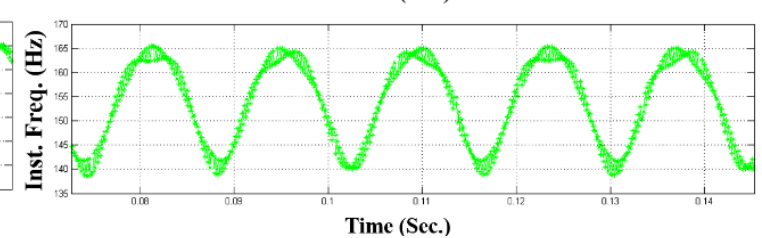

(d)

FIG. 8. Envelope and instantaneous frequency at the $20^{\text {th }}$ node: (a) combination of $101 \mathrm{~Hz}$ and $314 \mathrm{~Hz}$, (b) $164 \mathrm{~Hz}$ and $194 \mathrm{~Hz}$, (c) $74 \mathrm{~Hz}$ and $114 \mathrm{~Hz}$, (d) $154 \mathrm{~Hz}$ and $225 \mathrm{~Hz}$.

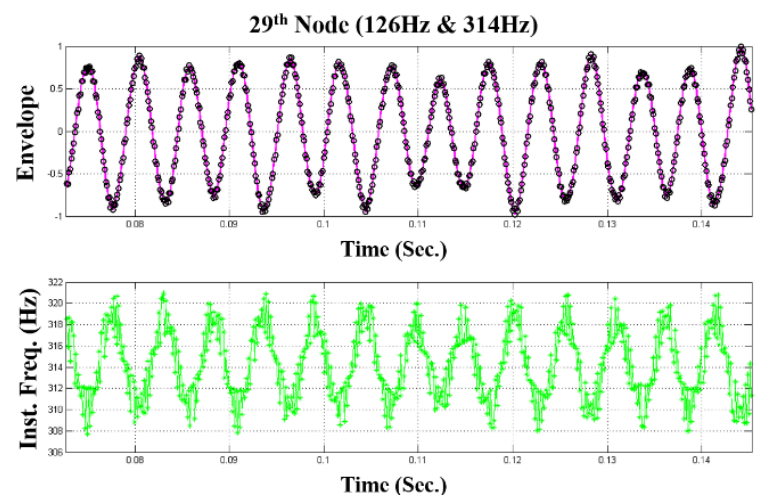

(a)
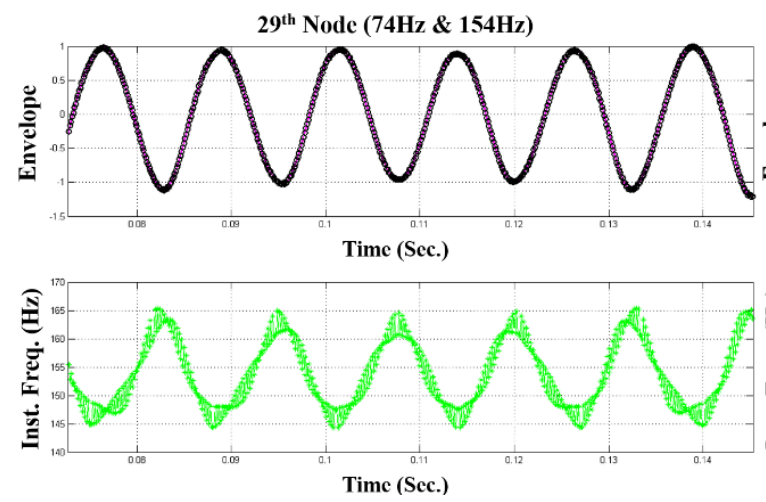

(c)

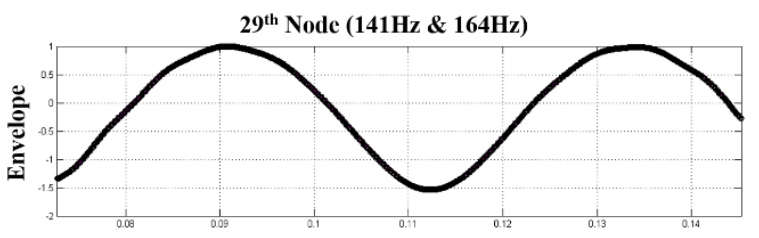

Time (Sec.)

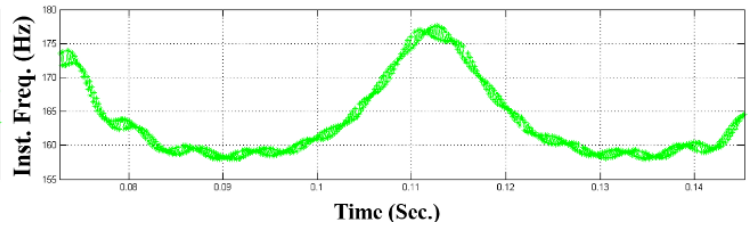

(b)
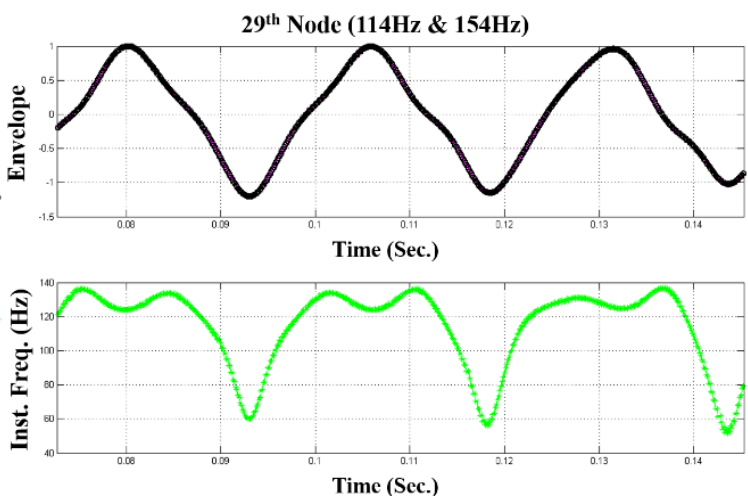

(d) 
FIG. 9. Envelope and instantaneous frequency at the $29^{\text {th }}$ node: (a) combination of $126 \mathrm{~Hz}$ and $314 \mathrm{~Hz}$, (b) $141 \mathrm{~Hz}$ and $164 \mathrm{~Hz}$, (c) $74 \mathrm{~Hz}$ and $154 \mathrm{~Hz}$, (d) $114 \mathrm{~Hz}$ and $154 \mathrm{~Hz}$.

The results in Figures 8 and 9 can be compared with the results from the pair waves that constitute the forward and backward waves in the same mode number. In Figure 8, part (a) is relevant to the distortion of an envelope and instantaneous frequency (case (1) \&(2)). Part (b) shows a fewer number of waves in the envelope than the expected mode number at $164 \mathrm{~Hz}$ and $194 \mathrm{~Hz}$ and it is also relevant to the distortion in the instantaneous frequency (zigzag) (case (2)\&(3)). Part (c) shows a fewer number of waves in the envelope than the frequency of interest at $114 \mathrm{~Hz}$ (case (3)). Part (d) is relevant to the distortion in the instantaneous frequency (zigzag) (case (2)). In Figure 9, part (a) is relevant to the distortion in the envelope and instantaneous frequency (case (1) \&(2)). Part (b) shows a fewer number of waves in the envelope than the expected mode number at $141 \mathrm{~Hz}$ and $164 \mathrm{~Hz}$, and it is also relevant to the distortion in the instantaneous frequency (zigzag) (case (2)\&(3). Part (c) is relevant to the distortion in the instantaneous frequency (zigzag) (case (2)). Part (d) is relevant to the distortion in the envelope and instantaneous frequency (case (1) \&(2)). The ambiguous wave combination can be distinguished from the truly matched pair of waves by using these reasons. Although there would be more possible combinations with ambiguous waves in addition to the example presented here, they would also be associated with any of the three cases. Figures 10 and 11 show the results of truly matched waves as the forward and backward that are estimated from Table II.
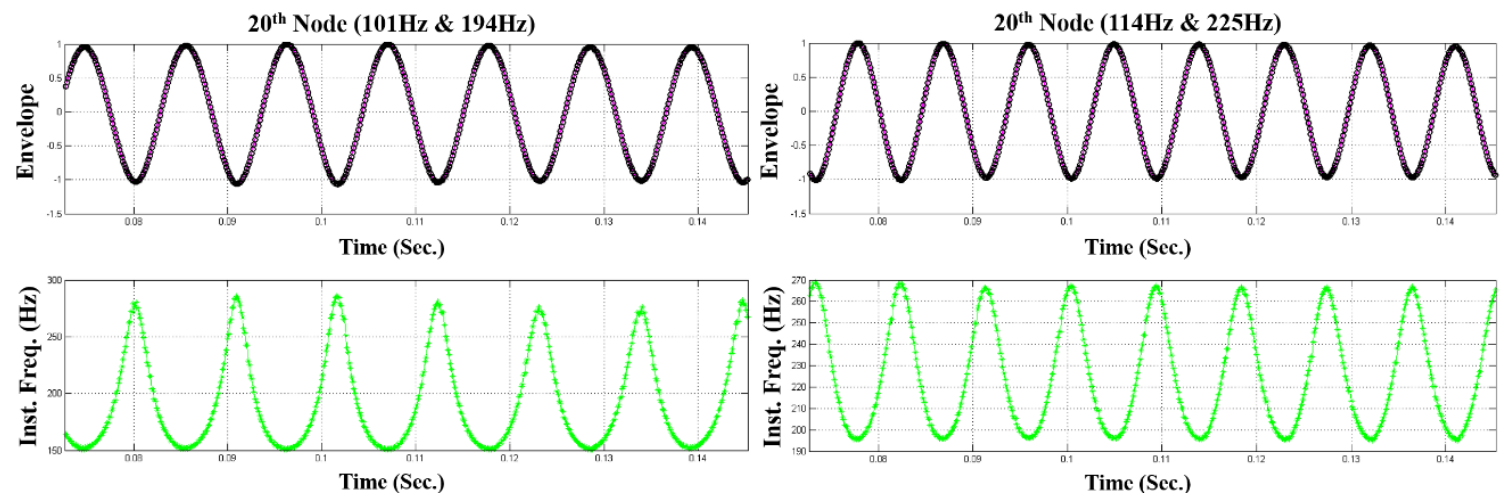
(a)

(b)

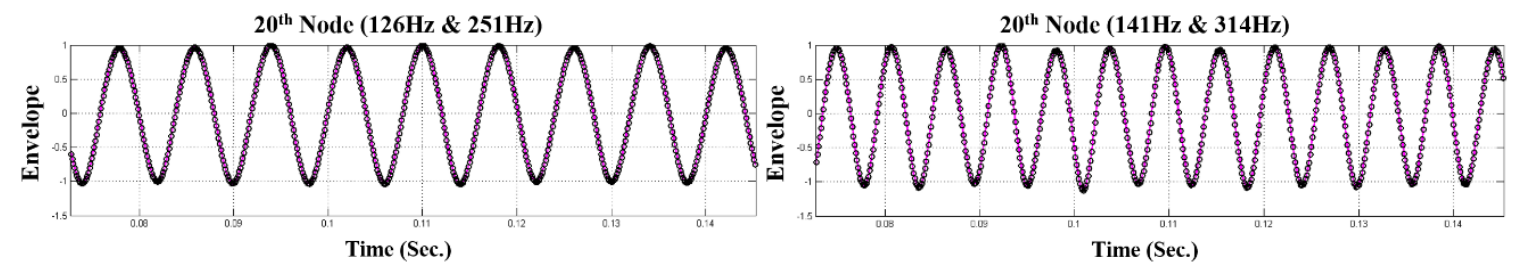

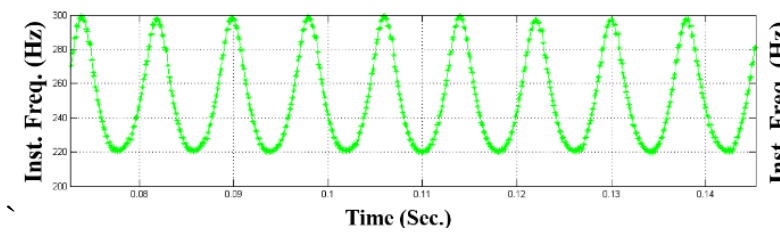

(c)

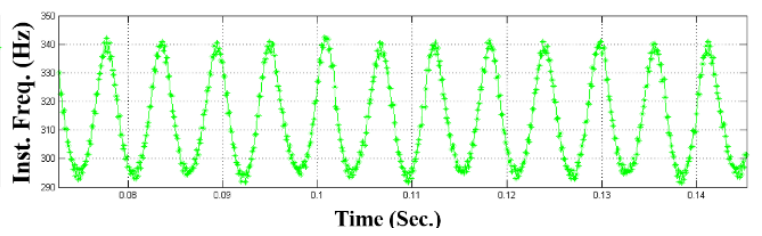

(d)

FIG. 10. Envelope and instantaneous frequency at the $20^{\text {th }}$ node, and combinations of the pair frequencies (a) $101 \mathrm{~Hz}$ and $194 \mathrm{~Hz}$, (b) $114 \mathrm{~Hz}$ and $225 \mathrm{~Hz}$, (c) $126 \mathrm{~Hz}$ and $251 \mathrm{~Hz}$, (d) $141 \mathrm{~Hz}$ and $314 \mathrm{~Hz}$.
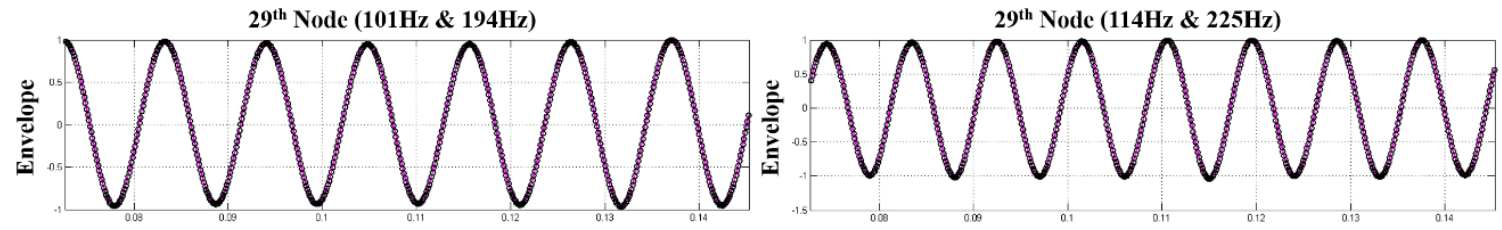

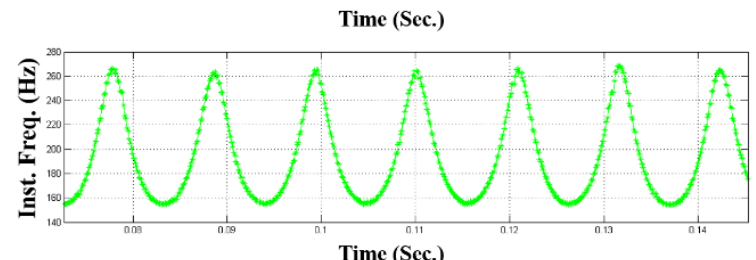

(a)

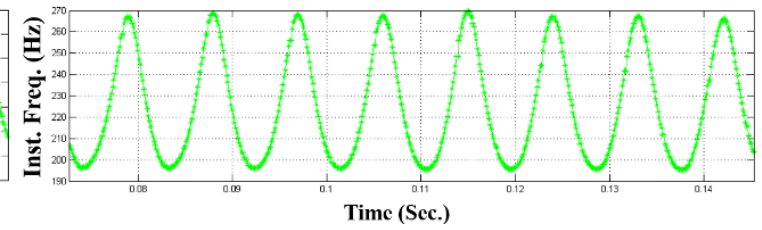

(b)

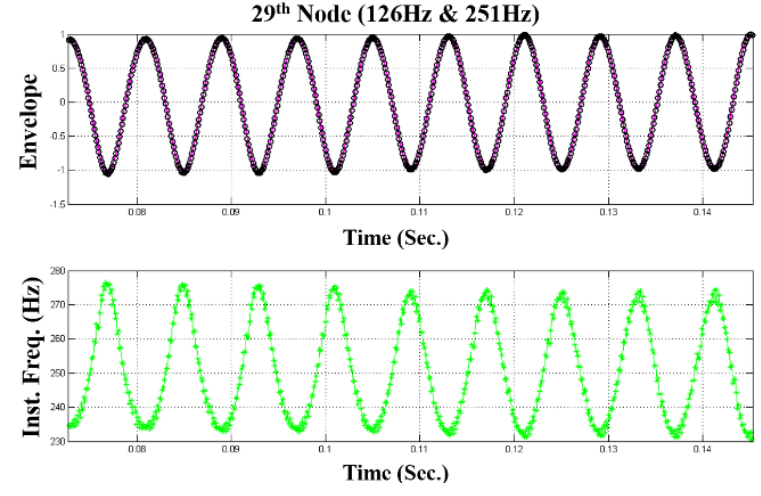

(c)

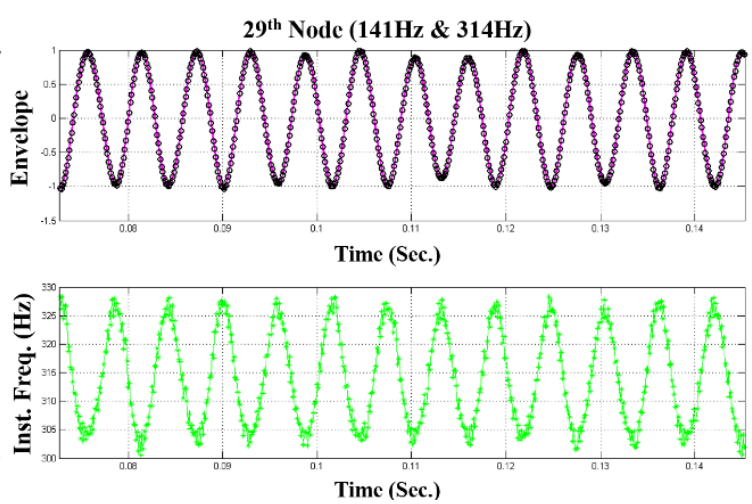

(d) 
FIG. 11. Envelope and instantaneous frequency at the $29^{\text {th }}$ node, and combinations of the pair frequencies (a) $101 \mathrm{~Hz}$ and $194 \mathrm{~Hz},(\mathrm{~b}) 114 \mathrm{~Hz}$ and $225 \mathrm{~Hz}$, (c) $126 \mathrm{~Hz}$ and $251 \mathrm{~Hz}$, (d) $141 \mathrm{~Hz}$ and $314 \mathrm{~Hz}$.

The results of Figures 10 and 11 are not associated with the three cases introduced previously. This result means that there is no distortion in the envelope and instantaneous frequency. Moreover, the mode number estimated using the obtained envelope is suitable at the combined frequencies. In addition, the envelopes in these two figures show differences in phase but have the same wave number for each mode number. Therefore, the pairs identified from Table II can also be identified using the HHT technique. Here, as shown in the previous figures, the obtained results are represented continuously. Therefore, the values at the position corresponding to 45 nodes (in circumferential coordinates, from $315^{\circ}$ (or $-45^{\circ}$ ) to $225^{\circ}$ by $6^{\circ}$ increments) are selected, and the selected values are represented in cylindrical coordinates in contrast to the example in Figure 1 (mode shape was represented in Cartesian coordinates).
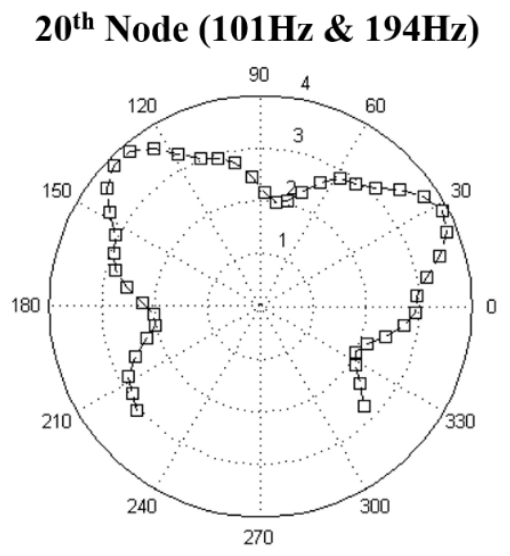

(a)
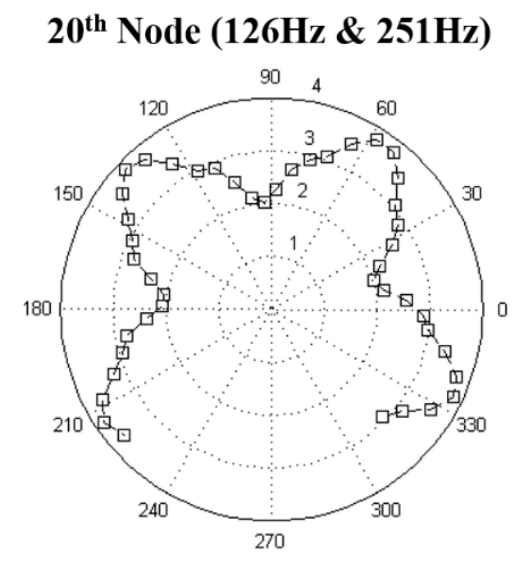
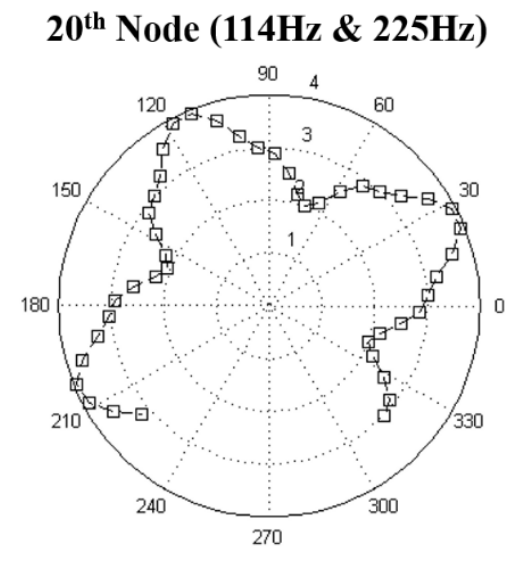

(b)

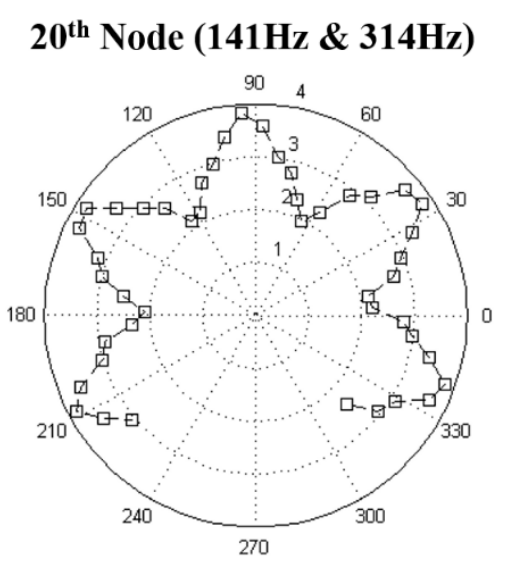


FIG. 12. Reconstructed mode shapes by the HHT at the $20^{\text {th }}$ node measurement using a combination of (a) $101 \mathrm{~Hz}$ and $194 \mathrm{~Hz}$, (b) $114 \mathrm{~Hz}$ and $225 \mathrm{~Hz}$, (c) $126 \mathrm{~Hz}$ and $251 \mathrm{~Hz}$, (d) $141 \mathrm{~Hz}$ and $314 \mathrm{~Hz}$.

$29^{\text {th }}$ Node $(101 \mathrm{~Hz} \& 194 \mathrm{~Hz})$

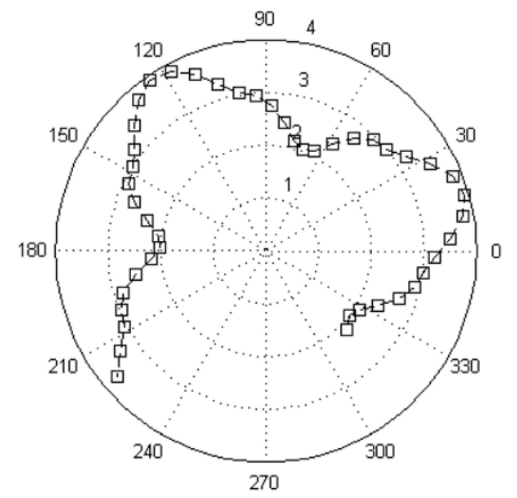

(a)

$29^{\text {th }}$ Node $(126 \mathrm{~Hz} \& 251 \mathrm{~Hz})$

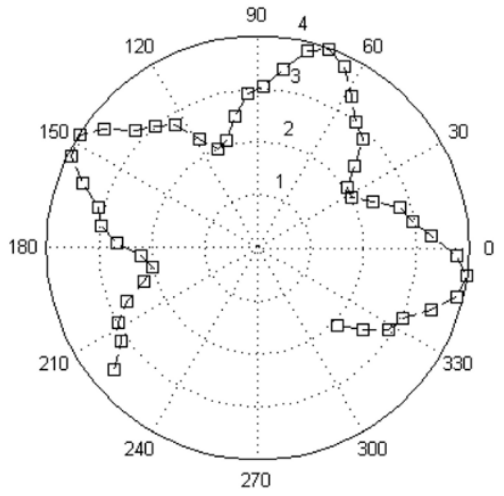

(c) $2^{\text {th }}$ Node $(114 \mathrm{~Hz} \& 225 \mathrm{~Hz})$

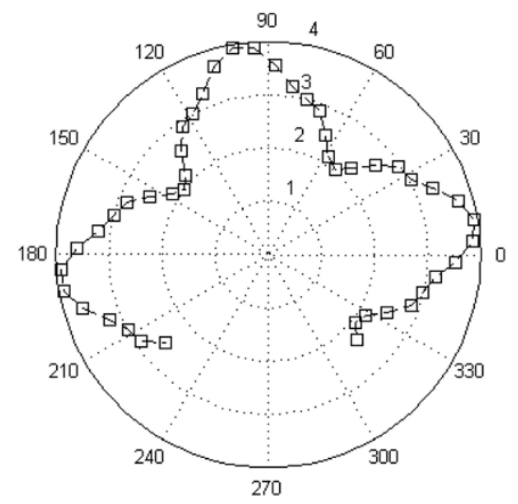

(b)
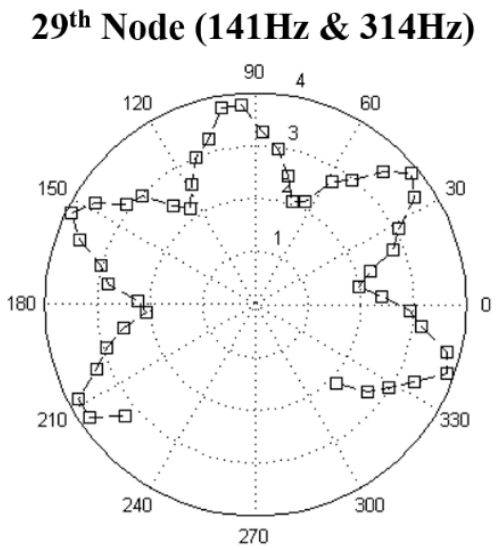

(d)

FIG. 13. Reconstructed mode shapes by the HHT at the $29^{\text {th }}$ node measurement using a combination of (a) $101 \mathrm{~Hz}$ and $194 \mathrm{~Hz}$, (b) $114 \mathrm{~Hz}$ and $225 \mathrm{~Hz}$, (c) $126 \mathrm{~Hz}$ and $251 \mathrm{~Hz}$, (d) $141 \mathrm{~Hz}$ and $314 \mathrm{~Hz}$.

The mode shapes in Figures 12 and 13 look similar to each other, but they have slightly different phase. Differences are observed between the reconstructed results obtained using the HHT and the measured results obtained from the 45 nodes (Figure 6). Therefore, the results of Figures 12 and 13 should be compared to the results of Figure 6 quantitatively. The results using the HHT, however, are represented by real values (i.e., standing wave), while the results of Figure 6, which propagate in 
the forward and backward directions, are represented by complex values as expressed in Equation (6b) (i.e., a traveling wave). Therefore, the results of Figures 12 and 13 should be transformed so that their rotating direction can be identified. First, one should assign a proper direction to each wave that appears as a pair in every plot in these figures. As in previous research studies, the resonance frequency of the backward wave has a larger magnitude than the forward wave in local coordinates. While, as explained in Refs. 15, 17 and in section III, the resonance frequencies are shifted in the negative frequency direction by an amount that is equal to the rotational speed multiplied by mode number. Therefore, the resonance frequency of the forward wave that is represented by the negative value has a larger magnitude than the resonance frequency of the backward wave that is represented by the positive value (refer to Figure 14). Therefore, in the two frequencies of each pair in Figures 12 and 13, the larger frequency represents the forward wave, whereas the smaller frequency represents the backward wave (e.g., in Figures 12 and 13 (a), 101-Hz mode indicates the backward wave, the 194-Hz mode indicates the forward wave). Based on Equation (6b), the traveling wave has a $90^{\circ}$ phase difference between the real and imaginary parts, and this phase variance can be obtained by the Hilbert transform.

$$
\begin{aligned}
& \phi_{\text {Reconstructed Backward }}=\phi_{H H T}-j H\left\{\phi_{H H T}\right\} \\
& \phi_{\text {Reconstructed Forward }}=\phi_{H H T}+j H\left\{\phi_{H H T}\right\}
\end{aligned}
$$

In Equation (19), $\phi_{H H T}$ represents the mode shape reconstructed from the HHT using two waves as a pair, which is represented by real value only, and $H$ indicates the Hilbert transform. $\phi_{\text {Reconstructed Forward }}$ and $\phi_{\text {Reconstructed Backward }}$ represent the reconstructed forward and backward traveling mode shapes, respectively. The results of Figure 12 (20 $0^{\text {th }}$ node) are transformed by Equation (19) to assign each direction, and the mode-shape results that have their own direction obtained using the transformation can be compared to Figure 6 using the MAC value. The MAC value results are listed in Table III according to the forward and backward directions.

TABLE III. MAC values for backward and forward waves 


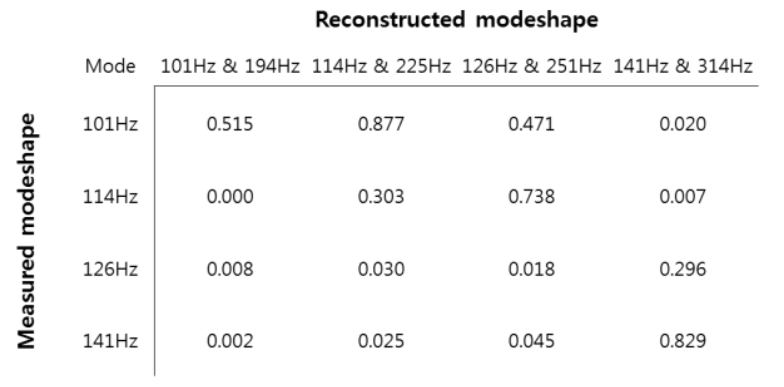

Backward Wave
Reconstructed modeshape

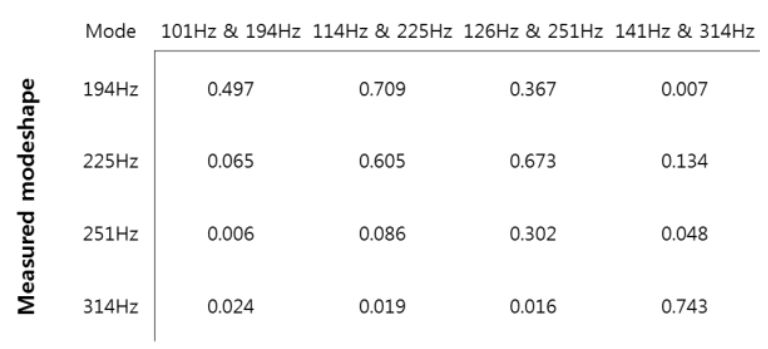

Forward Wave

If the two mode shapes that bear a similarity to one another are compared, then the diagonal elements in the MAC table will be close to the unit value, while the other values will be close to zero. However, the first elements in the upper triangle have a larger value than the corresponding values in the diagonal elements (e.g., for the backward-wave case: 0.877 [1, 2] and 0.738 $[2,3]$ and for the forward-wave case: $0.709[1,2]$ and $0.637[2,3])$. There are several reasons for this result. First, because of the characteristics of an optical sensor, it is difficult to avoid noise in the measurement whenever a laser measures a black object. To estimate the accurate modal parameters, the FRF, which is obtained by means of an applied force and response measurements, should be used, but in this study, only response measurements were used to estimate the dynamic characteristics of a rolling tire. In addition, as reported in a previous study ${ }^{13}$, a rolling tire has a different vibrational magnitude to the forcing position as a reference in the circumferential direction, but the mode shape reconstructed from the HHT has consistent magnitude in the circumferential direction. In addition, there are several reasons that account for these observations, but the most decisive reason is the Coriolis effect. In section II, a non-rotating system shows Doppler Effect in continuous measurement, but for the rotating case the Coriolis effect is also observed in continuous measurement as explained in section III. This effect can be described by following figure.

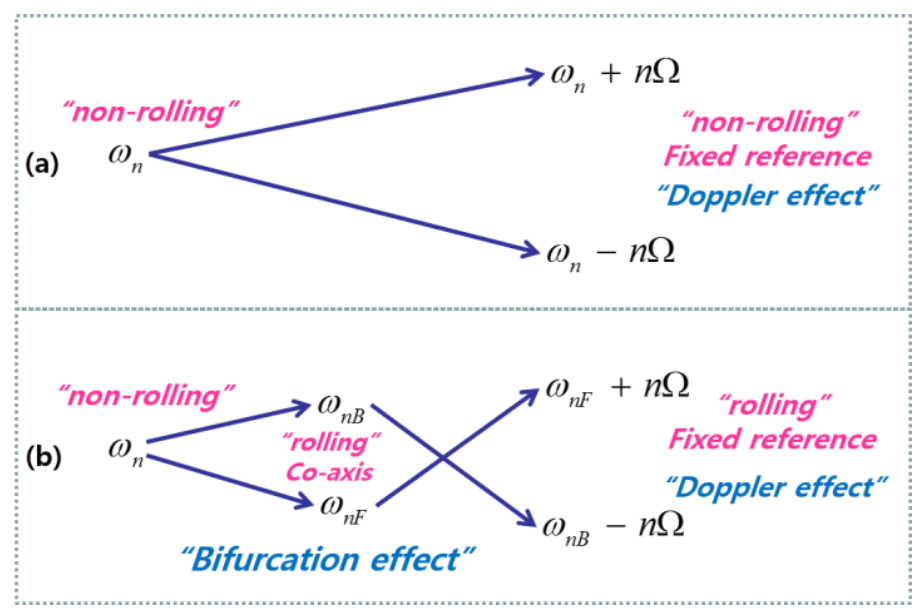


FIG. 14. The effect of natural frequency for continuous measurement (a) for the non-rotating case and (b) for the rotating case.

When we continuously measure a non-rotating structure, any given mode frequency $\omega_{n}$ is shifted in the positive and negative directions by an amount equal to the mode number multiplied by the scanning speed (scanning frequency). This shift results in a separation of this mode into two waves. The substitution of these two frequencies for each wave is represented by the mode number and scanning speed. For the rotating case, however, the given mode frequency $\omega_{n}$ is separated into two waves, $\omega_{n F}$ and $\omega_{n B}$, due to the Coriolis effect (bifurcation) in a co-rotating reference; the magnitudes of these two resultant frequencies are changed by an amount equal to the mode number multiplied by the scanning speed in the positive and negative directions, respectively, in the fixed reference. Therefore, the difference between these two resultant frequencies is not constituted by the mode number and the scanning speed only (i.e., the assumption of Equation (17) is wrong). Consequently, the magnitude of the difference between the two resultant frequencies is smaller for the rotating case than for the non-rotating case. Therefore, the last value of each column in the upper triangular elements shows larger values than the corresponding diagonal elements in Table III. This phenomenon, however, keeps decreasing as the mode number keeps increasing (e.g., for the backward wave: $0.829[4,4]>0.296[3,4])$. This phenomenon is natural because the resonance frequency at the higher mode is much larger than the rotating speed, and therefore, the effect of the rotating speed decreases at the higher mode. To conclude, the mode shape reconstructed using the HHT has a longer wavelength than the true mode shape at the frequency that has a relatively lower mode number compared to the rotating speed, but the results at the higher mode can be reliable.

In summary, the proposed method requires the existence of a pair of waves in the measurement, and the process to determine the presence of a pair of waves would require much effort. In addition, it is difficult to trust the reliability of the obtained results for modes that have a smaller mode frequency value than rotating speed. The possibility of confirming the modal parameters (forward, backward mode shape) of a rolling tire with only a single measurement has many advantages in terms of the difficulties, time consumption and economic aspects of the experimental setup for a rolling tire in spite of the drawbacks previously mentioned.

\section{CONCLUSION}


The HHT that is used for the continuous measurement of a non-rotating system to reconstruct the mode shape is adapted for a rolling tire measurement. To this end, a brief procedure for extracting the mode shape by means of the HHT is introduced, and the physical meaning of each stage is included. A rolling tire is assumed to be a flexible rotating model, as in previous studies. The numerical configuration of FRF for this model is derived, and the relationship between FRF and HHT is discussed. The required condition (i.e., a pair of forward and backward waves) to apply the proposed method for a rolling tire measurement is introduced, and the method to confirm this condition is proposed. In addition, ambiguous cases that can appear in the process

of confirming this condition with experimental measurements is introduced, and 3 characteristics that can make it possible to distinguish the case of the combination of two different directional waves in the same mode number with the ambiguous case are introduced. The proposed method is applied to the experimental measurements, and the obtained results are quantitatively compared with the results obtained from the conventional method, which requires several measurements to obtain the mode shape.

\section{ACKNOWLEDGEMENTS}

The authors would like to thank the EU Seventh Framework Program (FP7/2010) for its support under the TIRE-DYN project (grant agreement no 251211). The first author received financial support from the Dasan project and LMS International for the research stay in Leuven. He also gratefully thanks Prof. Chong-Won Lee for his technical advice in this research. This work was partially supported by a National Research Foundation of Korea (NRF) grant funded by the Korean government (2014R1A2A1A01005264).

\section{REFERENCES}

${ }^{1}$ P. Kindt, P. Sas and W. Desmet, J. Sound Vib. 326, 85-869 (2009).

${ }^{2}$ P. Kindt, P. Sas and W. Desmet, Opt. Laser Eng. 47, 443-453 (2009).

${ }^{3}$ P. Kindt, D. Berckmans, F. D. Coninck, P. Sas and W. Desmet, Mech. Syst. Signal Process. 23, 2557-2574 (2009).

${ }^{4}$ J. Lee, S. Wang, P. Kindt, B. Pluymers and W. Desmet, Int. J. Appl. Mech. (DOI: 10.1142/S1758825114500549 2014).

${ }^{5}$ N. E. Huang, Z. Shen, S. R. Long, M. C. Wu, H. H. Shih, Q. Zheng, N.-C. Yen, C. C. Tung, and H. H. Liu, Proc. R. Soc. London, Ser. A 454, 903-995 (1998). 
${ }^{6}$ M. Kang, A. Stanbridge, T. Chang and H. Kim, Mech. Syst. Signal Process. 16(2-3), 201-210 (2002).

${ }^{7}$ Y. Kyong, D. Kim, J. Dayou, K. Park, and S. Wang, Rev. Sci. Instrum. 79, 075103 (2008).

${ }^{8}$ J. Lee, H. S. Hussain, S. Wang and K. Park, Rev. Sci. Instrum. (doi: 10.1063/1.4894260 2014).

${ }^{9}$ D. J. Inman, Engineering Vibration (Prentice-Hall, New Jersey, 2001).

${ }^{10}$ S. Gong, A study of In-Plane Dynamics of Tires, (Ph.D. Thesis. TU Delft 1993).

${ }^{11}$ J. Lee, S. Wang, B. Pluymers, W. Desmet and P. Kindt, Mech. Syst. Signal Process (2014 (accepted)).

${ }^{12}$ C.-W. Lee, Mech. Syst. Signal Process. 5((2)), 119-137 (1991).

${ }^{13}$ Y. -J. Kim and J. S. Bolton, J. Sound Vib. 275, 605-621 (2004).

${ }^{14} \mathrm{P}$. Zegelaar, The dynamic response of tyres to brake torque variations and road undevennesses, (Ph.D. Thesis. TU Delft. 1998).

${ }^{15}$ P. Kindt, Structure-borne tyre/road noise due to road surface discontinuities, (Ph.D. thesis Leuven 2009).

${ }^{16}$ W. Heylen, S. Lammens, \& Sas, P. Modal Analysis Theory and Testing. K. U. Leuven - PMA (1998).

${ }^{17}$ S. Vercammen, C. González Díaz, P. Kindt, J. Middelberg, C. Thiry and J. Leyssens, International Conference on Noise and Vibration Engineering. Leuven. (2012). 